\title{
Chaotification for a Class of Delay Difference Equations Based on Snap-Back Repellers
}

\author{
Zongcheng Li, ${ }^{1,2}$ Shutang Liu, ${ }^{1}$ Wei Li, ${ }^{3}$ and Qingli Zhao ${ }^{2}$ \\ ${ }^{1}$ College of Control Science and Engineering, Shandong University, Jinan, Shandong 250061, China \\ ${ }^{2}$ School of Science, Shandong Jianzhu University, Jinan, Shandong 250101, China \\ ${ }^{3}$ Department of Public Foundation, Shandong Radio and TV University, Jinan, Shandong 250010, China
}

Correspondence should be addressed to Zongcheng Li; chengzi_0905@163.com

Received 29 April 2015; Accepted 12 July 2015

Academic Editor: Xinguang Zhang

Copyright (C) 2015 Zongcheng Li et al. This is an open access article distributed under the Creative Commons Attribution License, which permits unrestricted use, distribution, and reproduction in any medium, provided the original work is properly cited.

\begin{abstract}
We study the chaotification problem for a class of delay difference equations by using the snap-back repeller theory and the feedback control approach. We first study the stability and expansion of fixed points and establish a criterion of chaos. Then, based on this criterion of chaos and the feedback control approach, we establish a chaotification scheme such that the controlled system is chaotic in the sense of both Devaney and Li-Yorke when the parameters of this system satisfy some mild conditions. For illustrating the theoretical result, we give some computer simulations.
\end{abstract}

\section{Introduction}

Research on chaos control has attracted a lot of interest from many scientists and mathematicians. There are two directions in chaos control, that is, control of chaos and anticontrol of chaos (or called chaotification). The former regarded chaos as harmful. So many earlier works focused on stabilizing a chaotic system, which was regarded as the traditional control. The reader is referred to the monographs [1-3] for more details. However, in recent years, it has been found that chaos can actually be very useful in some applications; a typical example is chaos-based cryptography [4]. Hence, sometimes it is useful and even important to make a nonchaotic system chaotic, or to make a chaotic system produce a stronger or different type of chaos. This progress is called chaotification or anticontrol of chaos.

In research on chaotification for discrete dynamical systems, a mathematically rigorous and effective chaotification method was first proposed by Chen and Lai [5-7], where they first used the feedback control technique. This method plays an important role in studying chaotification problems of discrete dynamical systems. For a survey on chaotification of discrete dynamical systems, one can see [8] and some references therein.
To the best of our knowledge, although there already exist many works on chaotification of discrete dynamical systems, there are few results on chaotification of delay difference equations. Motivated by the feedback control approach, we have succeeded in studying the chaotification problems on linear delay difference equations [9] and a class of delay difference equations [10]. In the two papers, we use the sine functions as controllers to establish some chaotification schemes. The reason of using this type of controllers is that the sine function has some favorable properties and this designed controller is also simple, cheap, and implementable in real engineering applications (see [8-10] and the references therein). In the chaotification theorem of [10], the delay difference equations need to have at least two fixed points. However, there are also many delay difference equations with only one fixed point, which cannot satisfy the above condition. This motivates us to study this case. In this paper, we will apply the feedback control approach and the snapback repeller theory to study chaotification for a class of delay difference equations with at least one fixed point.

This paper is organized as follows. In Section 2, we give some basic concepts and one lemma. In Section 3, we study the stability and expansion of fixed points and establish a criterion of chaos. Based on this criterion of chaos, we 
establish a chaotification scheme for a class of delay difference equations with at least one fixed point. Then, we give some computer simulations to illustrate the theoretical result. Finally, we conclude this paper in Section 4.

\section{Preliminaries}

Up to now, there is no unified definition of chaos in mathematics. For convenience, we present two definitions of chaos, which will be used in this paper.

Definition 1 (see [11]). Let $(X, d)$ be a metric space, let $F$ : $X \rightarrow X$ be a map, and let $S$ be a set of $X$ with at least two distinct points. Then $S$ is called a scrambled set of $F$ if, for any two different points $x, y \in S$,

$$
\begin{gathered}
\liminf _{n \rightarrow \infty} d\left(F^{n}(x), F^{n}(y)\right)=0, \\
\limsup _{n \rightarrow \infty} d\left(F^{n}(x), F^{n}(y)\right)>0 .
\end{gathered}
$$

The map $F$ is said to be chaotic in the sense of Li-Yorke if there exists an uncountable scrambled set $S$ of $F$.

Remark 2. The term "chaos" was first used by Li and Yorke [12] for a map on a compact interval. Following the work of $\mathrm{Li}$ and Yorke, Zhou [11] gave the above definition of chaos for a topological dynamical system on a general metric space.

Definition 3 (see [13]). Let $(X, d)$ be a metric space. A map $F: V \subset X \rightarrow V$ is said to be chaotic on $V$ in the sense of Devaney if

(i) $F$ is topologically transitive in $V$;

(ii) the periodic points of $F$ are dense in $V$;

(iii) $F$ has sensitive dependence on initial conditions in $V$.

Remark 4. In [14], Huang and Ye showed that chaos in the sense of Devaney is stronger than that in the sense of Li-Yorke under some conditions.

The following criterion of chaos is established by Shi et al., which plays an important role in the present paper.

Lemma 5 (see [15, Theorem 2.1]; [16, Theorem 4.4]). Let $F$ : $\mathbf{R}^{n} \rightarrow \mathbf{R}^{n}$ be a map with a fixed point $z \in \mathbf{R}^{n}$. Assume that

(i) $F$ is continuously differentiable in a neighborhood of $z$ and all the eigenvalues of $\mathrm{DF}(z)$ have absolute values larger than 1, which implies that there exist a positive constant $r$ and a norm $\|\cdot\|$ in $\mathbf{R}^{n}$ such that $F$ is expanding in $\bar{B}_{r}(z)$ in $\|\cdot\|$, where $\bar{B}_{r}(z)$ is the closed ball of radius $r$ centered at $z$ in $\left(\mathbf{R}^{n},\|\cdot\|\right)$;

(ii) $z$ is a snap-back repeller of $F$ with $F^{m}\left(x_{0}\right)=z, x_{0} \neq$ $z$, for some $x_{0} \in B_{r}(z)$ and some positive integer $m$, where $B_{r}(z)$ is the open ball of radius $r$ centered at $z$ in $\left(\mathbf{R}^{n},\|\cdot\|\right)$. Furthermore, $F$ is continuously differentiable in some neighborhoods of $x_{0}, x_{1}, \ldots, x_{m-1}$, respectively, and $\operatorname{det} D F\left(x_{j}\right) \neq 0$ for $0 \leq j \leq m-1$, where $x_{j}=$ $F\left(x_{j-1}\right)$ for $1 \leq j \leq m-1$.
Then for each neighborhood $U$ of $z$, there exist a positive integer $k>m$ and a Cantor set $\Lambda \subset U$ such that $F^{k}: \Lambda \rightarrow \Lambda$ is topologically conjugate to the symbolic dynamical system $\sigma$ : $\Sigma_{2}^{+} \rightarrow \sum_{2}^{+}$. Consequently, there exists a compact and perfect invariant set $V \subset \mathbf{R}^{n}$, containing the Cantor set $\Lambda$, such that $F$ is chaotic on $V$ in the sense of Devaney as well as in the sense of Li-Yorke and has a dense orbit in $V$.

Remark 6. In 1978, Marotto [17] first gave the concept of snapback repeller for maps in $\mathbf{R}^{n}$. Later, in 2004, Shi and Chen [18] extended this concept to general metric spaces. According to the classifications of snap-back repellers for maps in metric spaces in [18], the snap-back repeller given by Marotto [17] is regular and nondegenerate. For more details on snap-back repeller, we refer to [16-19] and the references therein. We can easily conclude that the point $z$ in Lemma 5 is a regular and nondegenerate snap-back repeller. Hence, Lemma 5 can be summed as a single word: "a regular and nondegenerate snap-back repeller in $\mathbf{R}^{n}$ implies chaos in the sense of both Devaney and Li-Yorke." For more details, one can see $[15,16]$.

\section{Chaotification Based on Snap-Back Repellers}

In this paper, we will study the chaotification problem of a delay difference equation, chaotic or not, in the form of

$$
x(n+1)=f(x(n-k), x(n)), \quad n \geq 0,
$$

where $k \geq 1$ is a fixed integer and $f: D \subset \mathbf{R}^{2} \rightarrow \mathbf{R}$ is a map. Equation (2) is a discrete analogue of many one-dimensional delay differential equations, such as the well known MackeyGlass equation.

The objective here is to design a control input sequence $\{v(n)\}$ such that the output of the controlled system

$$
x(n+1)=f(x(n-k), x(n))+v(n), \quad n \geq 0,
$$

is chaotic in the sense of both Devaney and Li-Yorke. In our earlier paper [10], by using the result that heteroclinic cycles connecting repellers imply chaos established in [20], we have studied the chaotification problem of (2) for the case where (2) has at least two fixed points. However, there are also many delay discrete dynamical systems which only have one fixed point. Then, the chaotification scheme established in [10] cannot be used. In this paper, we will study the chaotification problem for the case where (2) has at least one fixed point. We design the controller as follows:

$$
v(n)=\alpha \operatorname{saw}_{\varepsilon}(\beta x(n-k)),
$$

where $\varepsilon>0$ is any given constant, $\alpha$ and $\beta$ are two undetermined parameters, and $\operatorname{saw}_{\varepsilon}(\cdot)$ is the classical sawtooth function; that is,

$$
\begin{aligned}
\operatorname{saw}_{\varepsilon}(x)=(-1)^{m} & (x-2 m \varepsilon), \\
& (2 m-1) \varepsilon \leq x<(2 m+1) \varepsilon, m \in \mathbf{Z},
\end{aligned}
$$

while $\mathbf{Z}$ denotes the integer set. Many researchers have succeeded in using the sawtooth function as a controller 
to chaotify discrete dynamical systems (see $[15,21]$ and the references therein).

Set

$$
u_{j}(n):=x(n+j-k-1), \quad 1 \leq j \leq k+1, n \geq 0 .
$$

Then (2) and the controlled system (3) with controller (4) can be transformed into the following $k+1$-dimensional discrete systems on $\mathbf{R}^{k+1}$ :

$$
\begin{aligned}
& u(n+1)=F(u(n)), \\
& u(n+1)=G(u(n)),
\end{aligned}
$$

respectively, where $u=\left(u_{1}, u_{2}, \ldots, u_{k+1}\right)^{T} \in \mathbf{R}^{k+1}$ and the maps $F, G: \mathbf{R}^{k+1} \rightarrow \mathbf{R}^{k+1}$.

As defined in [10], the maps $F$ and $G$ are called the maps induced by $f$ and $g$, respectively, where $g(x, y):=f(x, y)+$ $\alpha \operatorname{saw}_{\varepsilon}(\beta x)$. Systems (7) and (8) are called the systems induced by (2) and (3) in the Euclidean space $\mathbf{R}^{k+1}$, respectively. System (3) is said to be chaotic in the sense of Devaney (or Li-Yorke) on $V \subset \mathbf{R}^{k+1}$ if its induced system (8) is chaotic in the sense of Devaney (or Li-Yorke) on $V \subset \mathbf{R}^{k+1}$.

In the following, without loss of generality and for simplicity, we can suppose that the origin $O:=(0, \ldots, 0)^{T} \in \mathbf{R}^{k+1}$ is always a fixed point of the induced system (7). Otherwise, if none of the fixed points is the origin $O$, then we can choose a transformation of coordinates such that one of the fixed points becomes the origin $O$ in a new coordinate system. Then the map $f$ in (2) satisfies $f(0,0)=0$, throughout the rest of the paper.

It is well known that the stability and expansion of a map at a fixed point has a close relationship with the modulus of the eigenvalues of its derivative operator when the map is differentiable at the fixed point. Suppose that $f$ is differentiable at $(0,0)$; then the induced map $F$ is differentiable at $O$. Let $f_{x}(x, y)$ and $f_{y}(x, y)$ denote the first partial derivatives of $f$ with respect to the first and the second variables at the point $(x, y)$, respectively. Then we can get the following results on stability and expansion of the fixed point $O$ of the induced system (7).

Theorem 7. Assume that $k<\infty$. Denote $a:=f_{y}(0,0), b:=$ $f_{x}(0,0)$.

(i) If $f$ is differentiable at $(0,0)$, then, for $a=0$, the fixed point $O$ of system (7) is asymptotically stable if and only if $|b|<1$; and for $a \neq 0$, the fixed point $O$ of system (7) is asymptotically stable if and only if $|a|<(k+1) / k$, and

$$
\begin{aligned}
|a|-1 & <-b<\left(a^{2}+1-2|a| \cos \phi\right)^{1 / 2}, \quad \text { for kodd, } \\
|a+b| & <1, \\
|b| & <\left(a^{2}+1-2|a| \cos \phi\right)^{1 / 2},
\end{aligned}
$$

$$
\text { for } k \text { even, }
$$

where $\phi$ is the solution in $(0, \pi /(k+1))$ of equation $\sin (k \theta) / \sin [(k+1) \theta]=1 /|a|$. (ii) If $f$ is continuously differentiable in a neighborhood of $(0,0)$ and $|b|-|a|>1$, then the fixed point $O$ of system (7) is a regular expanding fixed point in some norm in $\mathbf{R}^{k+1}$.

Proof. When $a=0$, it is easy to obtain that all the eigenvalues of $D F(O)$ have absolute values less than 1 if and only if $|b|<$ 1. So, the result in (i) holds. When $a \neq 0$, the result in (i) can be directly derived by using Theorem 3 in [22]. Result (ii) can be derived from Lemma 2.1 of [10]. This completes the proof.

Now, we establish a criterion of chaos for the induced system (7).

Theorem 8. Let $f: D \subset \mathbf{R}^{2} \rightarrow \mathbf{R}$ be a map and let it be continuously differentiable in a neighborhood of $(0,0)$ with $f(0,0)=0$. Assume that

(i) $\left|f_{x}(0,0)\right|-\left|f_{y}(0,0)\right|>1$, which implies that there exist a positive constant $r^{*}$ and a norm $\|\cdot\|^{*}$ in $\mathbf{R}^{k+1}$ such that $F$ is continuously differentiable in $\bar{B}_{r^{*}}(O)$ and $O$ is a regular expanding fixed point of $F$ in $\bar{B}_{r^{*}}(O)$ in the norm $\|\cdot\|^{*}$, where $\bar{B}_{r^{*}}(O)$ is the closed ball of radius $r^{*}$ centered at $O$ in $\left(\mathbf{R}^{k+1},\|\cdot\|^{*}\right)$;

(ii) there exists a point $u^{*} \in D$ with $u^{*} \neq 0$, such that $f$ is continuously differentiable in a neighborhood of $\left(u^{*}, 0\right)$ with $f\left(u^{*}, 0\right)=0, f_{x}\left(u^{*}, 0\right) \neq 0$,

(iia) when $k=1$, there exist $x_{1}, x_{2} \in\left(-r^{*}, r^{*}\right)$ such that $x_{1}^{2}+x_{2}^{2} \neq 0,\left(x_{1}, x_{2}\right)^{T} \in B_{r^{*}}(O), f$ is continuously differentiable in a neighborhood of $\left(x_{2}, u^{*}\right)$, and

$$
\begin{gathered}
f\left(x_{2}, u^{*}\right)=0, \\
f\left(x_{1}, x_{2}\right)=u^{*}, \\
f_{x}\left(x_{2}, u^{*}\right) \neq 0, \\
f_{x}\left(x_{1}, x_{2}\right) \neq 0 ;
\end{gathered}
$$

(iib) when $k>1$, there exist $x_{1}, x_{2} \in\left(-r^{*}, r^{*}\right)$ such that $x_{1}^{2}+x_{2}^{2} \neq 0,\left(x_{1}, x_{2}, 0, \ldots, 0\right)^{T} \in B_{r^{*}}(O), f$ is continuously differentiable in a neighborhood of $\left(x_{2}, u^{*}\right)$, and

$$
\begin{gathered}
f\left(x_{2}, u^{*}\right)=0, \\
f\left(x_{1}, 0\right)=u^{*}, \\
f_{x}\left(x_{2}, u^{*}\right) \neq 0, \\
f_{x}\left(x_{1}, 0\right) \neq 0 .
\end{gathered}
$$

Then the induced system (7), and consequently system (2), is chaotic in the sense of both Devaney and Li-Yorke.

Proof. We will apply Lemma 5 to prove this theorem. So, we only need to show that all the assumptions in Lemma 5 are satisfied. 
It follows from assumption (i) and the second conclusion of Theorem 7 that $F$ is continuously differentiable in $\bar{B}_{r^{*}}(O)$, all the eigenvalues of $D F(O)$ have absolute values larger than 1 , and $O$ is a regular expanding fixed point of $F$ in $\bar{B}_{r^{*}}(O)$ in some norm $\|\cdot\|^{*}$ of $\mathbf{R}^{k+1}$. Therefore, condition (i) in Lemma 5 is satisfied.

Next, we will show that $O$ is a snap-back repeller of $F$ in the norm $\|\cdot\|^{*}$. In the following, we will show that there exists a point $O_{0} \in W$ with $O_{0} \neq O$ satisfying

$$
F^{k+2}\left(O_{0}\right)=O
$$

which implies that $O$ is a snap-back repeller of $F$.

For the case where $k=1$, it follows from condition (iia) that there exists a point $O_{0}=\left(x_{1}, x_{2}\right)^{T} \in B_{r^{*}}(O), O_{0} \neq O$, such that $O_{1}=F\left(O_{0}\right)=\left(x_{2}, u^{*}\right)^{T}, O_{2}=F^{2}\left(O_{0}\right)=\left(u^{*}, 0\right)^{T}$, and $F^{3}\left(O_{0}\right)=O$.

For the case where $k>1$, it follows from condition (iib) that there exists a point $O_{0}=\left(x_{1}, x_{2}, 0, \ldots, 0\right)^{T} \in B_{r^{*}}(O)$, $O_{0} \neq O$, such that $O_{1}=F\left(O_{0}\right)=\left(x_{2}, 0, \ldots, 0, u^{*}\right)^{T}, O_{j}=$ $F^{j}\left(O_{0}\right)=(0, \ldots, 0, \underbrace{u^{*}, 0, \ldots, 0}_{j})^{T}$ for $2 \leq j \leq k+1$, and $F^{k+2}\left(O_{0}\right)=O$.

It is obvious that $F$ is continuously differentiable in some neighborhoods of $O_{j}:=F\left(O_{j-1}\right)$ for $1 \leq j \leq k+1$. So, we need to show that the following holds:

$$
\operatorname{det} D F\left(O_{j}\right) \neq 0, \quad 0 \leq j \leq k+1 .
$$

If $F$ is differentiable at $u=\left(u_{1}, \ldots, u_{k+1}\right)^{T} \in \mathbf{R}^{k+1}$, then a direct calculation shows that

$$
\operatorname{det} D F(u)=(-1)^{k} f_{x}\left(u_{1}, u_{k+1}\right) .
$$

From condition (i), we get that $\left|f_{x}(0,0)\right|>1+\left|f_{y}(0,0)\right|>$ 0 , which together with condition (ii) and (14) implies that conclusion (13) holds for $k=1$ and $k>1$.

Therefore, all the assumptions in Lemma 5 are satisfied. Then the induced system (7), and consequently system (2), is chaotic in the sense of both Devaney and Li-Yorke. This completes the proof.

Remark 9. Since $f$ is a function of two variables, the conditions in (ii) of Theorem 8 are not very strict conditions.

Based on Theorem 8, a chaotification scheme for the controlled system (3) with controller (4) is established in the following.

Theorem 10. Consider the controlled system (3) with controller (4). Assume that

(i) $f$ is continuously differentiable in $[-r, r]^{2}$ for some $r>0$ with $f(0,0)=0$, which implies that there exist positive constants $M$ and $N$ such that for any $(x, y) \in$ $[-r, r]^{2}$

$$
\begin{aligned}
& |f(x, y)| \leq M \\
& \left|f_{x}(x, y)\right| \leq N \\
& \left|f_{y}(x, y)\right| \leq N
\end{aligned}
$$

(ii) there exists a point $u^{*} \in(-r, r)$ with $u^{*} \neq 0$ such that $f\left(u^{*}, 0\right)=0$.

Then there exist two positive constants $\alpha_{0}$ and $\beta_{0}$ satisfying

$$
\begin{aligned}
& \alpha_{0}>\frac{M+\left|u^{*}\right|}{\varepsilon}, \\
& \beta_{0}:=\frac{2 m_{0} \varepsilon}{u^{*}}>\max \left\{\frac{1+2 N}{\alpha_{0}}, \frac{3 \varepsilon}{r}\right\},
\end{aligned}
$$

where $\varepsilon>0$ is any given constant and $m_{0}$ is some integer, such that, for any $\alpha>\alpha_{0}$ and $\beta=\beta_{0}$, the controlled system (3) with controller (4) is chaotic in the sense of both Devaney and Li-Yorke.

Proof. We will use Theorem 8 to prove this theorem. So, it suffices to show that the map $g(x, y):=f(x, y)+\alpha \operatorname{saw}_{\varepsilon}(\beta x)$ satisfies all the assumptions in Theorem 8 .

For convenience, let $\alpha>\alpha_{0}, \beta=2 m \varepsilon / u^{*}>$ $\max \left\{(1+2 N) / \alpha_{0}, 3 \varepsilon / r\right\}$ throughout the proof, where $m$ is an undetermined integer. Let $G$ denote the induced map of $g$.

It is obvious that the function $\operatorname{saw}_{\varepsilon}(\beta x)$ is continuously differentiable in $(-\varepsilon / \beta, \varepsilon / \beta)$. Then, from assumption (i), we obtain that $g$ is continuously differentiable in $(-\varepsilon / \beta, \varepsilon / \beta)^{2}$ with $g(0,0)=0, O$ is a fixed point of the map $G$, and $G$ is continuously differentiable in $(-\varepsilon / \beta, \varepsilon / \beta)^{k+1}$. It follows from the last two relations of (15) that

$$
\begin{aligned}
\left|g_{x}(0,0)\right| & =\left|f_{x}(0,0)+\alpha \beta\right| \geq \alpha \beta-\left|f_{x}(0,0)\right| \\
& \geq \alpha \beta-N>1+N \geq 1+\left|f_{y}(0,0)\right| \\
& =1+\left|g_{y}(0,0)\right| .
\end{aligned}
$$

So condition (i) in Theorem 8 holds. Consequently, there exist a positive constant $r^{*}$ and a norm $\|\cdot\|^{*}$ in $\mathbf{R}^{k+1}$ such that $G$ is continuously differentiable in $\bar{B}_{r^{*}}(O)$ and $O$ is a regular expanding fixed point of $G$ in $\bar{B}_{r^{*}}(O)$ in the norm $\|\cdot\|^{*}$, where $\bar{B}_{r^{*}}(O) \subset(-\varepsilon / \beta, \varepsilon / \beta)^{k+1}$ is the closed ball of radius $r^{*}$ centered at $O$ in $\left(\mathbf{R}^{k+1},\|\cdot\|^{*}\right)$. Further, suppose that $W \subset$ $B_{r^{*}}(O)$ is an arbitrary neighborhood of $O$ in $\mathbf{R}^{k+1}$. Then there exists a neighborhood $U$ of 0 such that $U \times U \times \cdots \times U \subset W$.

Next, we need to show that $g$ satisfies assumption (ii) in Theorem 8. It is obvious that $\operatorname{saw}_{\varepsilon}\left(\beta u^{*}\right)=0$ and $\operatorname{saw}_{\varepsilon}(\beta x)$ is continuously differentiable in a neighborhood of $u^{*}$. So, $g(x, y)$ is continuously differentiable in a neighborhood of $\left(u^{*}, 0\right)$. From assumption (ii) and condition (15), it follows that

$$
\begin{aligned}
g\left(u^{*}, 0\right) & =f\left(u^{*}, 0\right)+\alpha \operatorname{saw}_{\varepsilon}\left(\beta u^{*}\right)=0, \\
\left|g_{x}\left(u^{*}, 0\right)\right| & =\left|f_{x}\left(u^{*}, 0\right)+(-1)^{m} \alpha \beta\right| \\
& \geq \alpha \beta-\left|f_{x}\left(u^{*}, 0\right)\right|>1+N>0 .
\end{aligned}
$$

For $k=1$, let

$$
h_{1}(x):=f\left(x, u^{*}\right)+\alpha \operatorname{saw}_{\varepsilon}(\beta x) .
$$


It follows from assumption (i) and the definition of sawtooth function that $h_{1}$ is continuous in $[-\varepsilon / \beta, 3 \varepsilon / \beta]$. From the first relation of (15), we get that

$$
\begin{gathered}
h_{1}\left(\frac{\varepsilon}{\beta}\right)=f\left(\frac{\varepsilon}{\beta}, u^{*}\right)+\alpha \varepsilon \geq \alpha \varepsilon-M>0, \\
h_{1}\left(\frac{3 \varepsilon}{\beta}\right)=f\left(\frac{3 \varepsilon}{\beta}, u^{*}\right)-\alpha \varepsilon \leq M-\alpha \varepsilon<0 .
\end{gathered}
$$

Therefore, by the intermediate value theorem, there exists a point $x_{2}$ with $\varepsilon / \beta<x_{2}<3 \varepsilon / \beta$, such that $h_{1}\left(x_{2}\right)=0$; that is, $g\left(x_{2}, u^{*}\right)=0$. Similarly, let

$$
h_{2}(x):=f\left(x, x_{2}\right)+\alpha \operatorname{saw}_{\varepsilon}(\beta x)-u^{*} .
$$

It is also clear that $h_{2}$ is continuous in $[-\varepsilon / \beta, 3 \varepsilon / \beta]$. It also follows from the first relation of (15) that

$$
\begin{aligned}
h_{2}\left(\frac{\varepsilon}{\beta}\right) & =f\left(\frac{\varepsilon}{\beta}, x_{2}\right)+\alpha \varepsilon-u^{*} \geq \alpha \varepsilon-M-\left|u^{*}\right| \\
& >0 \\
h_{2}\left(\frac{3 \varepsilon}{\beta}\right) & =f\left(\frac{3 \varepsilon}{\beta}, x_{2}\right)-\alpha \varepsilon-u^{*} \leq M+\left|u^{*}\right|-\alpha \varepsilon \\
& <0 .
\end{aligned}
$$

By the intermediate value theorem again, there exists a point $x_{1}$ with $\varepsilon / \beta<x_{1}<3 \varepsilon / \beta$, such that $h_{2}\left(x_{1}\right)=0$; that is, $g\left(x_{1}, x_{2}\right)=u^{*}$. It is clear that $x_{1}$ and $x_{2}$ are both in $(\varepsilon / \beta, 3 \varepsilon / \beta)=\left(u^{*} \varepsilon / 2 m r, u^{*} \varepsilon / 2 m r\right)$. So we can take a sufficiently large integer $m_{1}>0$, such that $x_{1}, x_{2} \in U$ with $x_{1}^{2}+x_{2}^{2} \neq 0$, and $\left(x_{1}, x_{2}\right)^{T} \in W$ for any $|m| \geq m_{1}$. It can easily be proved that $g$ is continuously differentiable in some neighborhoods of $\left(x_{2}, u^{*}\right)$ and $\left(x_{1}, x_{2}\right)$. Now, we show $g_{x}\left(x_{2}, u^{*}\right) \neq 0$. Otherwise, if $g_{x}\left(x_{2}, u^{*}\right)=0$, then the following equality holds:

$$
f_{x}\left(x_{2}, u^{*}\right)+(-1)^{m} \alpha \beta=0 .
$$

Hence, $\alpha \beta=\left|f_{x}\left(x_{2}, u^{*}\right)\right| \leq N$, which is a contradiction. Similarly, we can prove that $g_{x}\left(x_{1}, x_{2}\right) \neq 0$. Hence, condition (iia) in Theorem 8 holds.

For $k>1$, the determination of $x_{2}$ can be derived from the proof of the above paragraph as $k=1$. That is, there exists a point $x_{2}$ in $(\varepsilon / \beta, 3 \varepsilon / \beta)$, such that $h_{1}\left(x_{2}\right)=0$; that is, $g\left(x_{2}, u^{*}\right)=0$. Set

$$
h_{3}(x):=f(x, 0)+\alpha \operatorname{saw}_{\varepsilon}(\beta x)-u^{*} .
$$

With a similar method to the above paragraph, we can also get that there exists a point $x_{1}$ in $(\varepsilon / \beta, 3 \varepsilon / \beta)$ such that $h_{3}\left(x_{1}\right)=$ 0 , which implies that $g\left(x_{1}, 0\right)=u^{*}$. So we can also take a sufficiently large integer $m_{2}>0$, such that $x_{1}, x_{2} \in U$ with $x_{1}^{2}+x_{2}^{2} \neq 0$, and $\left(x_{1}, x_{2}, 0, \ldots, 0\right)^{T} \in W$ for any $|m| \geq m_{2}$. It can also easily be proved that $g$ is continuously differentiable in some neighborhoods of $\left(x_{2}, u^{*}\right)$ and $\left(x_{1}, x_{2}\right)$. The proofs of $g_{x}\left(x_{2}, u^{*}\right) \neq 0$ and $g_{x}\left(x_{1}, 0\right) \neq 0$ are similar to the above paragraph. So, the details are omitted.
Finally, let $\left|m_{0}\right|=\max \left\{m_{1}, m_{2}\right\}$. Then condition (ii) in Theorem 8 is satisfied for $m=m_{0}$. Therefore, for any $\alpha>\alpha_{0}$ and $\beta=\beta_{0}$, the controlled system (3) with controller (4) is chaotic in the sense of both Devaney and Li-Yorke. The proof is complete.

Remark 11. It is clear that the classical sinusoidal function $\sin x$ has similar geometric properties to the sawtooth function. So the following function

$$
v(n)=\alpha \sin (\beta x(n-k))
$$

can also be used as a controller to chaotify system (2), where $\beta$ is some constant to be determined and $\alpha>0$ is the controlled parameter. In fact, with a similar argument to the proof of Theorem 10, one can show that there also exist two positive constants $\beta_{0}$ and $\alpha_{0}$ such that for any constant $\alpha>\alpha_{0}$ and $\beta=\beta_{0}$ the result in Theorem 10 holds.

Remark 12. In [10], a similar result is given for a class of maps with at least two fixed points. In such a case, the two chaotification schemes obtained in [10] and this paper can be used. However, there will be many chaotic invariant sets as pointed out in Lemma 2.2 of [10] when using the chaotification scheme in [10]. It seems that the chaotic behaviors induced by a heteroclinic cycle connecting repellers are more complex than that induced by a single snap-back repeller. The difference between them will be our further research. But when the original system only has one fixed point, the chaotification scheme obtained in [10] cannot be used. Then, we can use the chaotification scheme obtained in this paper to chaotify this system.

Remark 13. Since the point $u^{*}$ in assumption (ii) of Theorem 10 can be negative, the value of $m_{0}$ determined in this paper can be a negative integer. In addition, it is very difficult to determine the concrete value $m_{0}$ since the concrete expanding area of a fixed point is not easy to obtain. To the best of our knowledge, there are few methods to determine the concrete expanding area of a fixed point in the existing literatures. So, in practical problems, we can take $\left|m_{0}\right|$ large enough such that the chaotification scheme can be effective.

In the last part of this section, we give an example to illustrate the theoretical result of Theorem 10.

Example 14. We take the map $f$ in (2) as the following:

$$
f(x, y)=0.01 x(x-1)-0.01 y^{2} \text {. }
$$

It is clear that $f$ is continuously differentiable in $\mathbf{R}^{2}$ and satisfies $f(0,0)=0$. Without loss of generality, we take $r=3$ in Theorem 10. Then, for any $(x, y) \in[-3,3]^{2}$, we get that

$$
\begin{gathered}
|f(x, y)| \leq 0.21, \\
\left|f_{x}(x, y)\right| \leq 0.07, \\
\left|f_{y}(x, y)\right| \leq 0.06 .
\end{gathered}
$$

Hence, we take $M=0.21, N=0.07$, and $r=3$ in assumption (i) of Theorem 10. It is also clear that the equation $f(x, 0)=0$ 


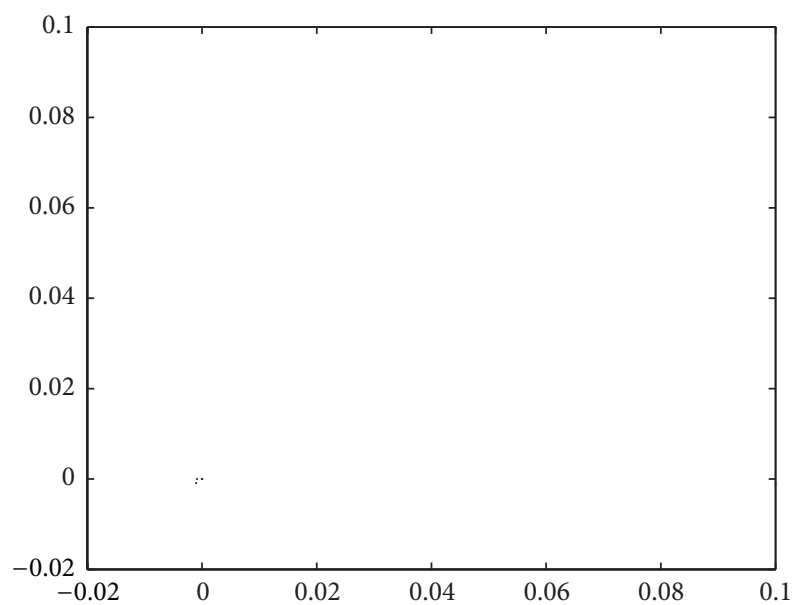

FIGURE 1: Simple dynamical behaviors of uncontrolled system (7) for $k=1$, where the initial value is taken as $u(0)=(0.1,0.1)^{T}$.

has a nonzero solution $u^{*}=1$, which lies in $(-3,3)$. Therefore, all the assumptions in Theorem 10 are satisfied. Here, we take the constant $\varepsilon=1$ in controller (4). Then, it follows from Theorem 10 that there exist two positive constants

$$
\begin{aligned}
\alpha_{0} & >\frac{M+\left|u^{*}\right|}{\varepsilon}=1.21, \\
\beta_{0} & =\frac{2 m_{0} \varepsilon}{u^{*}}=2 m_{0}>\max \left\{\frac{1+2 N}{\alpha_{0}}, \frac{3 \varepsilon}{r}\right\} \\
& =\max \left\{\frac{1.14}{\alpha_{0}}, 1\right\}=1,
\end{aligned}
$$

where $m_{0}$ is some positive integer, such that, for any $\alpha>\alpha_{0}$ and $\beta=\beta_{0}$, the controlled system (3) with controller (4) is chaotic in the sense of both Devaney and Li-Yorke.

In fact, there is only one fixed point $O:=(0, \ldots, 0)^{T} \in$ $\mathbf{R}^{k+1}$ in the uncontrolled system (7). It is obvious that $f_{y}(0,0)=0$ and $f_{x}(0,0)=-0.01$, which imply that $O$ is asymptotically stable from result (i) in Theorem 7 . It is also clear that all the solutions of the uncontrolled system (7) are bounded if the initial values are taken from $[-3,3]^{k+1}$. Therefore, if we take an initial condition $u(0)=(0.1, \ldots, 0.1)^{T} \epsilon$ $\mathbf{R}^{k+1}$, then the solution $u(n)$ of the uncontrolled system (7) should tend to the asymptotically stable fixed point $O$ when $n$ tends to infinity. This is confirmed in Figures 1 and 3.

Here, we take $\varepsilon=1, m_{0}=10, \alpha=30, \beta=20$, $k=1,2$, and $n$ from 0 to 20000 for computer simulations. The simulated results show that the original system (7) has simple dynamical behaviors, and the controlled system (8) has complex dynamical behaviors; see Figures 1-4.

It should be pointed out that the relative existing chaotification scheme in [10] is not available for this map since there is only one fixed point.

\section{Conclusion}

In this paper, we study the chaotification problem for a class of delay difference equations with at least one fixed

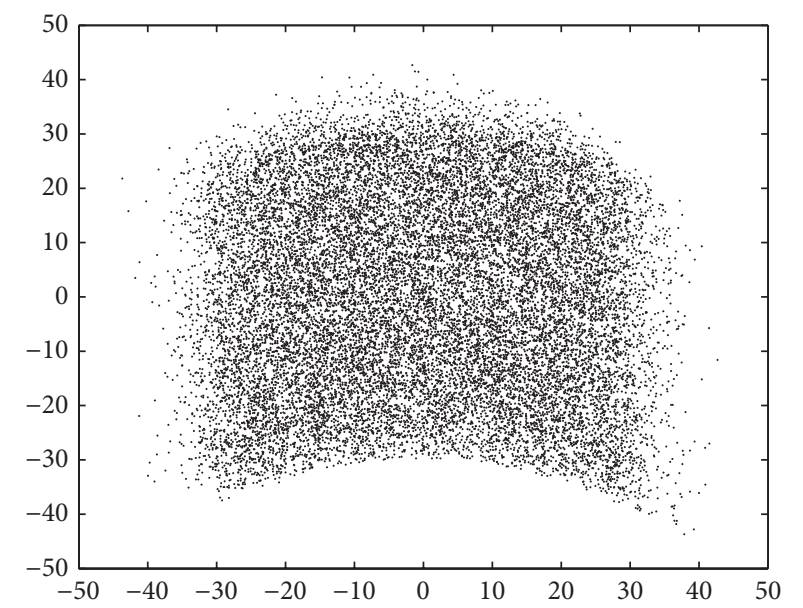

FIgURE 2: Complex dynamical behaviors of controlled system (8) for $\alpha=30, \beta=20, \varepsilon=1$, and $k=1$, where the initial value is taken as $u(0)=(0.1,0.1)^{T}$.

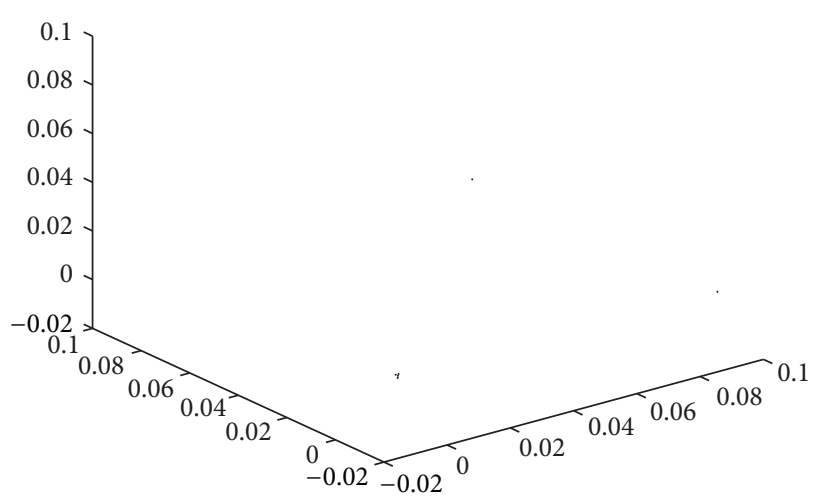

FIGURE 3: Simple dynamical behaviors of uncontrolled system (7) for $k=2$, where the initial value is taken as $u(0)=(0.1,0.1,0.1)^{T}$.

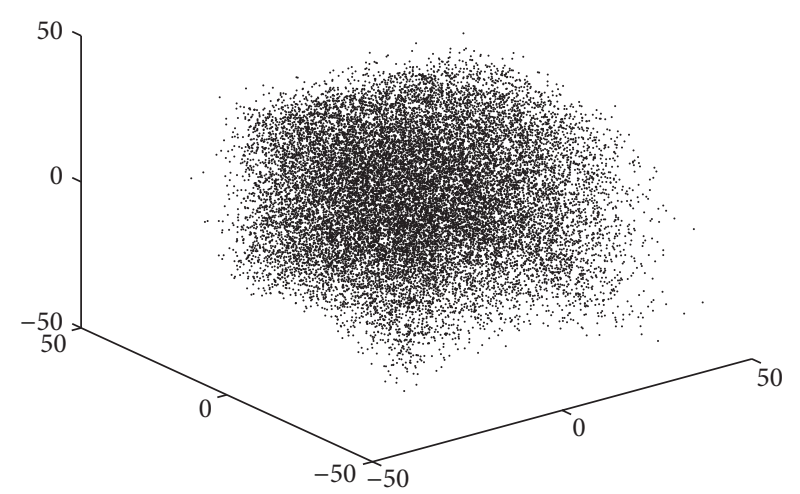

FIgURE 4: Complex dynamical behaviors of controlled system (8) for $\alpha=30, \beta=20, \varepsilon=1$, and $k=2$, where the initial value is taken as $u(0)=(0.1,0.1,0.1)^{T}$. 
point. We first establish a criterion of chaos by using the snap-back repeller theory. Then, based on this criterion of chaos and the feedback control approach, we establish a chaotification scheme. We have proved that the controlled system is chaotic in the sense of both Devaney and LiYorke when the parameters of the system satisfy some mild conditions. Numerical simulations confirm the theoretical analysis. The chaotification problem for more general maps in the original system will be our further research.

\section{Conflict of Interests}

The authors declare that there is no conflict of interests regarding the publication of this paper.

\section{Acknowledgments}

This work was supported by the National Natural Science Foundation of China (Grants 11101246, 61273088, and 10971120), the Nature Science Foundation of Shandong Province (Grant ZR2010FM010), and the Postdoctoral Science Foundation of China (Grant 2014M561908).

\section{References}

[1] G. R. Chen and X. N. Dong, From Chaos to Order: Methodologies, Perspectives, and Applications, World Scientific Publishing, Singapore, 1998.

[2] T. Kapitaniak, Chaos for Engineers: Theory, Applications, and Control, Springer, New York, NY, USA, 1998.

[3] A. L. Fradkov and A. Pogromsky, Introduction to Control of Oscillations and Chaos, World Scientific, Singapore, 1999.

[4] G. Jakimoski and L. Kocarev, "Chaos and cryptography: block encryption ciphers based on chaotic maps," IEEE Transactions on Circuits and Systems. I. Fundamental Theory and Applications, vol. 48, no. 2, pp. 163-169, 2001.

[5] G. R. Chen and D. J. Lai, "Feedback control of Lyapunov exponents for discrete-time dynamical systems," International Journal of Bifurcation and Chaos in Applied Sciences and Engineering, vol. 6, no. 7, pp. 1341-1349, 1996.

[6] G. R. Chen and D. J. Lai, "Anticontrol of chaos via feedback," in Proceedings of the IEEE Conference on Decision and Control, pp. 367-372, San Diego, Calif, USA, 1997.

[7] G. R. Chen and D. J. Lai, "Feedback anticontrol of discrete chaos," International Journal of Bifurcation and Chaos in Applied Sciences and Engineering, vol. 8, no. 7, pp. 1585-1590, 1998.

[8] G. R. Chen and Y. M. Shi, "Introduction to anti-control of discrete chaos: theory and applications," Philosophical Transactions of the Royal Society of London. Series A. Mathematical, Physical and Engineering Sciences, vol. 364, no. 1846, pp. 2433-2447, 2006.

[9] Z. C. Li, "Chaotification for linear delay difference equations," Advances in Difference Equations, vol. 2013, article 59, 11 pages, 2013.

[10] Z. C. Li, "Anticontrol of chaos for a class of delay difference equations based on heteroclinic cycles connecting repellers," Abstract and Applied Analysis, vol. 2014, Article ID 260150, 8 pages, 2014.

[11] Z. L. Zhou, Symbolic Dynamics, Shanghai Scientific and Technological Education Publishing House, Shanghai, China, 1997.
[12] T. Y. Li and J. A. Yorke, "Period three implies chaos," The American Mathematical Monthly, vol. 82, no. 10, pp. 985-992, 1975.

[13] R. L. Devaney, An Introduction to Chaotic Dynamical Systems, Addison-Wesley, New York, NY, USA, 1987.

[14] W. Huang and X. D. Ye, "Devaney's chaos or 2-scattering implies Li-Yorke's chaos," Topology and Its Applications, vol. 117, no. 3, pp. 259-272, 2002.

[15] Y. M. Shi, P. Yu, and G. R. Chen, "Chaotification of discrete dynamical systems in Banach spaces," International Journal of Bifurcation and Chaos in Applied Sciences and Engineering, vol. 16, no. 9, pp. 2615-2636, 2006.

[16] Y. M. Shi and G. R. Chen, "Discrete chaos in Banach spaces," Science in China Series A: Mathematics, vol. 34, pp. 595-609, 2004 (Chinese), English version: vol. 48, pp. 222-238, 2005.

[17] F. R. Marotto, "Snap-back repellers imply chaos in $R^{n}$," Journal of Mathematical Analysis and Applications, vol. 63, no. 1, pp. 199223, 1978.

[18] Y. M. Shi and G. R. Chen, "Chaos of discrete dynamical systems in complete metric spaces," Chaos, Solitons and Fractals, vol. 22, no. 3, pp. 555-571, 2004.

[19] F. R. Marotto, “On redefining a snap-back repeller," Chaos, Solitons and Fractals, vol. 25, no. 1, pp. 25-28, 2005.

[20] Z. C. Li, Y. M. Shi, and W. Liang, "Discrete chaos induced by heteroclinic cycles connecting repellers in Banach spaces," Nonlinear Analysis: Theory, Methods \& Applications, vol. 72, no. 2, pp. 757-770, 2010.

[21] X. F. Wang and G. R. Chen, "Chaotification via arbitrary small feedback controls," International Journal of Intelligent Systems, vol. 10, pp. 549-570, 2000.

[22] S. A. Kuruklis, "The asymptotic stability of $x_{n+1}-a x_{n}+b x_{n-k}=$ 0," Journal of Mathematical Analysis and Applications, vol. 188, no. 3, pp. 719-731, 1994. 


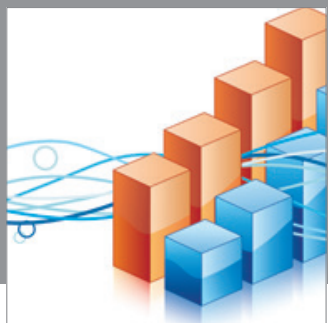

Advances in

Operations Research

mansans

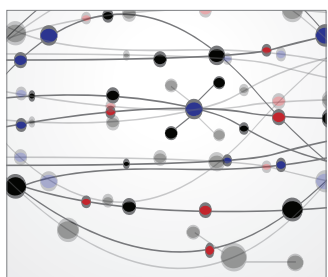

The Scientific World Journal
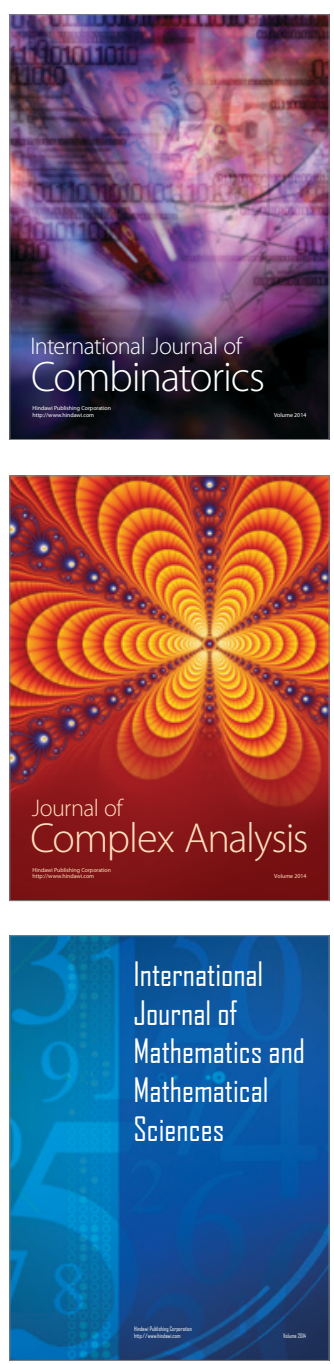
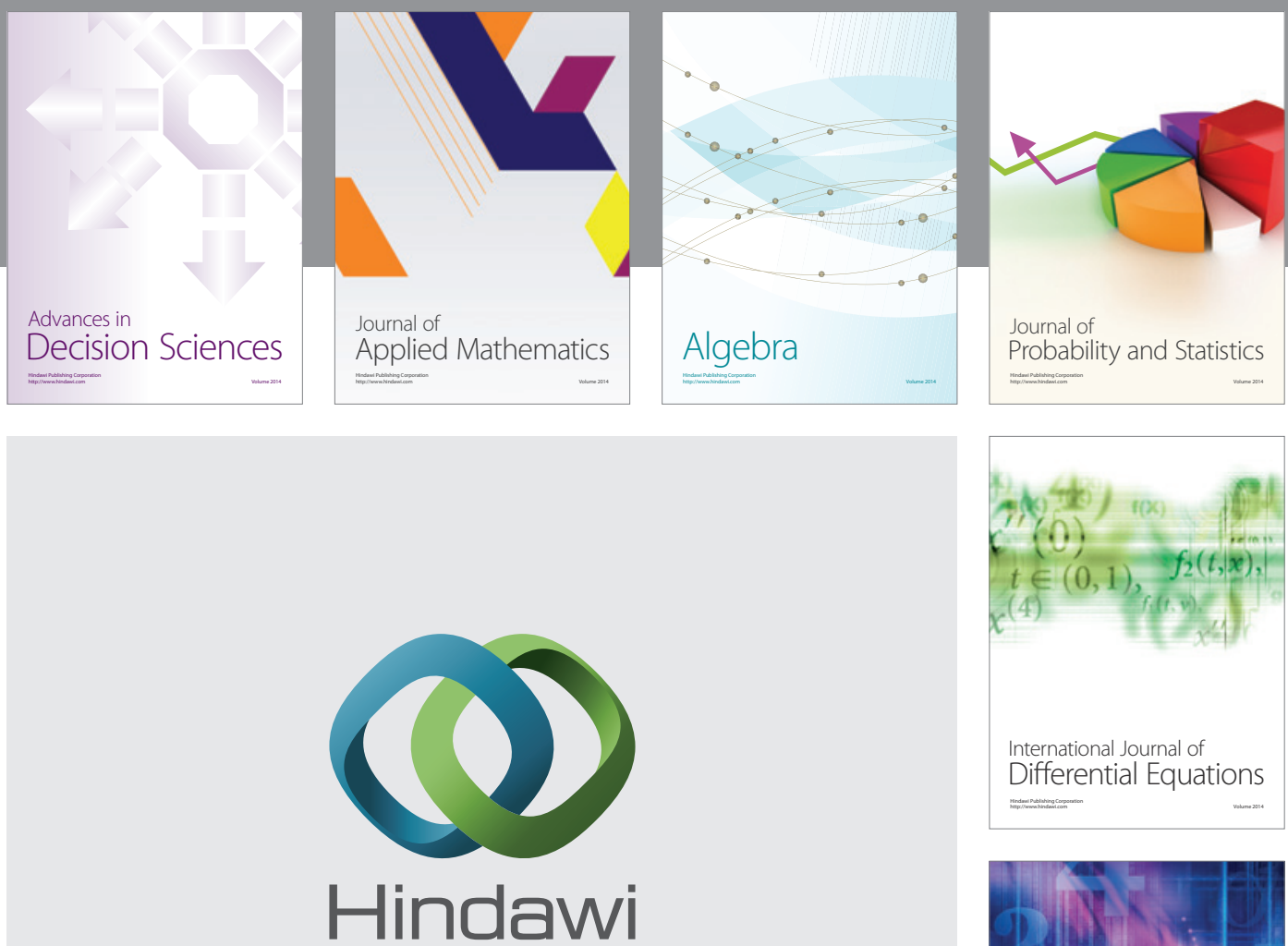

Submit your manuscripts at http://www.hindawi.com
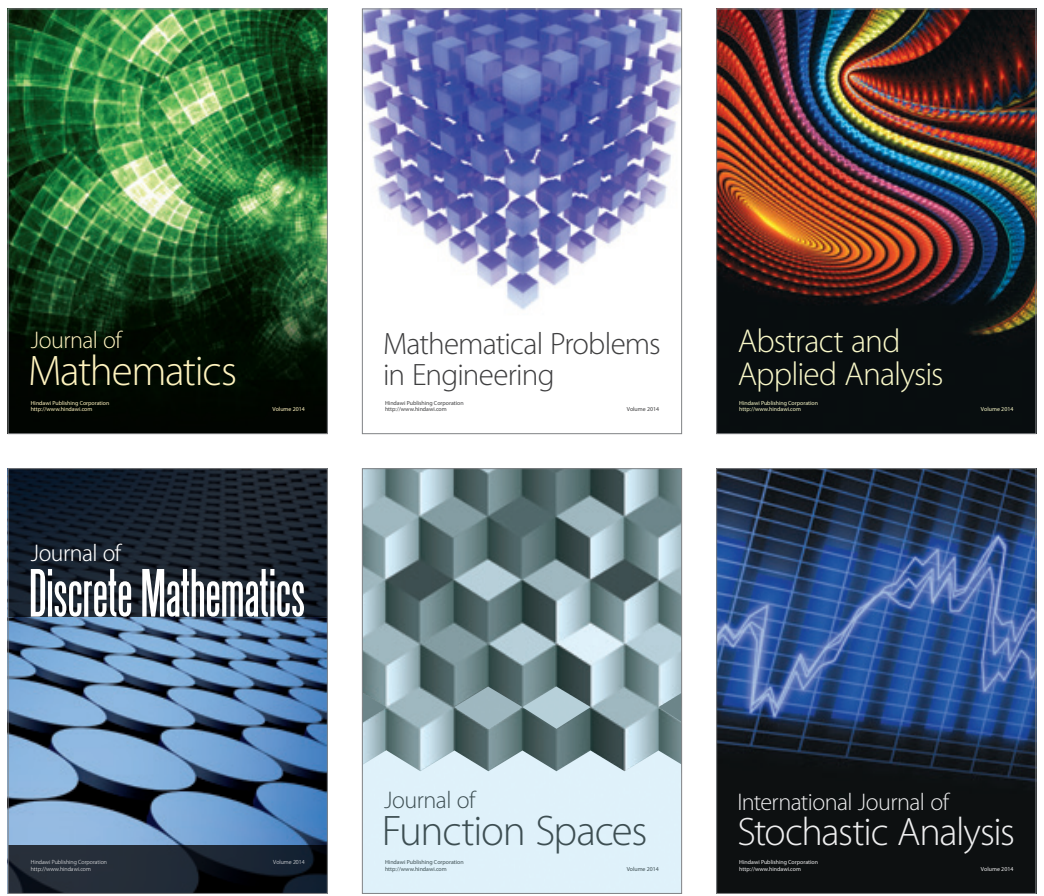

Journal of

Function Spaces

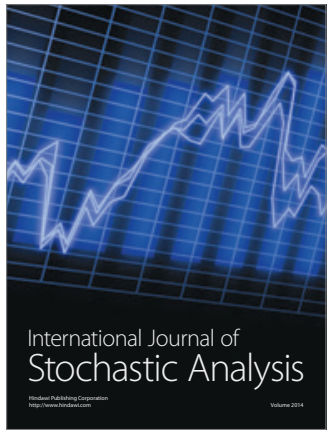

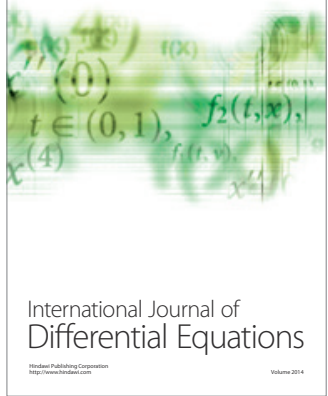
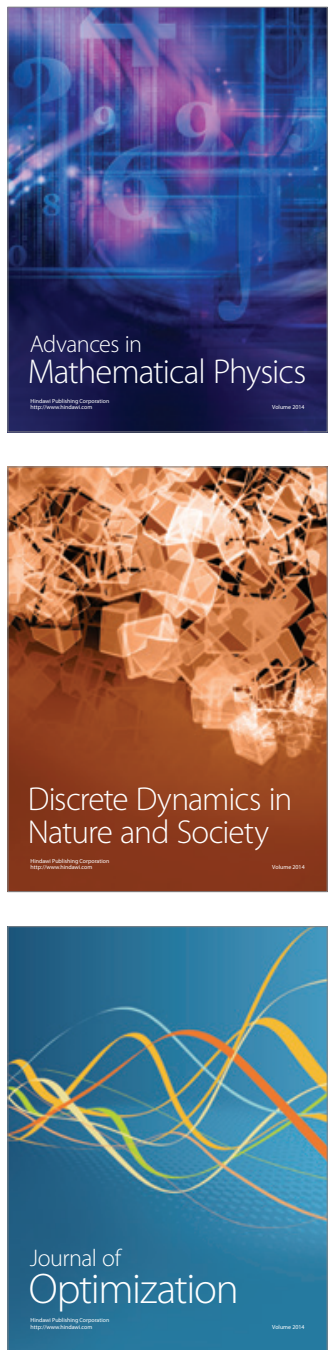